\title{
Gait mechanics contribute to exercise induced pain flares in knee osteoarthritis
}

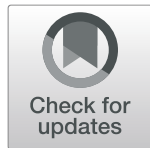

Katherine A. Boyer ${ }^{1,2,3^{*}}$ (D) and Jocelyn F. Hafer ${ }^{1,4}$

\begin{abstract}
Background: Exercise-induced pain flares represent a significant barrier for individuals with knee osteoarthritis to meet physical activity recommendations. There is a need to understand factors that contribute to pain flares and the potential for the motor system to adapt and reduce joint loading should a flare occur. The study aim was to examine the impact of a bout of exercise on self-reported pain, walking mechanics and muscle co-contraction for participants with knee osteoarthritis.
\end{abstract}

Methods: Thirty-six adults (17 healthy older and 19 knee osteoarthritis) participated in this study. Self-reported pain, joint mechanics and muscle co-activation during gait at two self-selected speeds were collected before and after a 20-min preferred pace treadmill walk (20MTW).

Results: Eight of nineteen osteoarthritis participants had a clinically significant pain flare response to the 20MTW. At baseline the participants that did not experience a pain flare had smaller knee flexion and total reaction moments compared to both the participants with pain flares $(p=0.02 ; p=0.05)$ and controls $(p<0.001 ; p<0.001)$. In addition, the 2nd peak knee adduction $(p=0.01)$ and internal rotation $(p=0.001)$ moments were smaller in the no flares as compared to controls. The pain flare participants differed from controls with smaller knee internal rotation moments $(p=0.03)$, but greater relative hamstrings (vs. quadriceps) and medial (vs. lateral) muscle activation ( $p=0.04, p=0.04)$ compared to both controls and no flare participants $(p=0.04, p=0.007)$. Following the 20MTW there were greater decreases in the 1 st and 2nd peak knee adduction ( $p=0.03 ; p=0.02)$, and internal rotation $(p=0.002)$ moments for the pain flare as compared to the no flare group. In addition, for the pain flare as compared to controls, greater decreases in the knee flexion $(p=0.03)$ and internal rotation $(p=0.005)$ moments were found.

Conclusions: Individuals who adapt their gait to reduce knee joint loads may be less susceptible to exercise-induced pain flares. This highlights a potential role of gait biomechanics in short-term osteoarthritis pain fluctuations. The results also suggest that despite the chronic nature of osteoarthritis pain, the motor system's ability to respond to nociceptive stimuli remains intact.

Keywords: Knee osteoarthritis, Gait mechanics, Exercise induced pain flare; muscle activation

\section{Background}

Knee osteoarthritis (OA) is one of the most common sources of musculoskeletal pain in adults over the age of 50 [1]. Pain and symptom management are the primary targets for clinical care of knee OA as there are no widely available disease modifying treatments. While use of analgesic and anti-inflammatory drugs is common, the primary

\footnotetext{
* Correspondence: kboyer@kin.umass.edu

'Department of Kinesiology, University of Massachusetts-Amherst, 110

Totman Building 30 Eastman Lane, Amherst, MA 01003, USA

${ }^{2}$ University of Massachusetts-Amherst, Mechanical and Industrial Engineering,

Amherst, USA

Full list of author information is available at the end of the article
}

recommendation for long term symptom management is exercise [2]. However, single bouts of weight-bearing exercise are known to acutely exacerbate pain, making movement-evoked pain flares (i.e., acute increases in pain) a characteristic symptom of knee OA [3-5]. These pain flares and the associated decreases in physical performance [6] can contribute to poor quality of life for individuals with knee OA. Pain flares also represent a major challenge to patient compliance with exercise prescription and programs, which is problematic as adherence to exercise training reduces pain flare magnitude over time $[2,5]$. As pain and variations in pain through-out a day remain a target

(c) The Author(s). 2019 Open Access This article is distributed under the terms of the Creative Commons Attribution 4.0 International License (http://creativecommons.org/licenses/by/4.0/), which permits unrestricted use, distribution, and reproduction in any medium, provided you give appropriate credit to the original author(s) and the source, provide a link to the Creative Commons license, and indicate if changes were made. The Creative Commons Public Domain Dedication waiver (http://creativecommons.org/publicdomain/zero/1.0/) applies to the data made available in this article, unless otherwise stated. 
for most OA treatments, determining the mechanisms at play in exercise induced pain is essential.

Gait biomechanics may play an important role in exercise induced pain flares. When compared to age-matched healthy adults, it's well documented that OA patients exhibit altered gait mechanics [7-9]. Within and between person variations in gait mechanics can alter muscle, external and soft tissue forces in the knee and may change the mechanical stimuli in joint tissue that would contribute to pain [10]. Initial cross sectional studies suggest a relationship between the knee adduction moment, a surrogate measure of the distribution of load between the medial and lateral compartments of the knee, and OA symptomatic and radiographic severity [8, 11-13]. Further, in a within patient analysis, a positive association between knees with pain on walking and the knee adduction moment magnitude during a subsequent walk has been reported [14]. Finally, when peak knee moments are reduced using shoe interventions, clinically relevant reductions in chronic joint pain have been reported [15]. Together these studies suggest that variations in gait mechanics, in particular the external knee flexion and adduction moments, can alter the pain experienced in knee OA, however whether greater moments might contribute to pain flares during exercise is not clear.

One of the challenges in quantifying the role of variations in gait mechanics on pain in OA is that gait mechanics may act as both a stimulus for pain and/or be altered as part of a motor system response to joint pain. The leading theories of pain-related movement adaptations suggest several neuromuscular responses that would lead to biomechanical adaptations such as increases in stiffness and decreases in joint movement and a reduction or redistribution of the total load on the painful joint [16-18]. In healthy young adults, acute pain induced by injection of hypertonic saline causes decreases in knee moments and quadriceps activation that are similar to gait adaptations seen with OA pain [19-21]. These studies provide evidence of the nature of the biomechanical response to knee joint pain however, the application of these studies' findings to knee OA may be limited because gait and pain response may differ between young adults and adults who are more similar in age to individuals with knee OA. Given the negative impact of intermittent pain on physical performance there is a need to understand if and how individuals modify their biomechanics in response to increases in knee joint pain. Elucidating this response is necessary to understand both the mechanisms and targets for management of changes in performance with intermittent pain.

Typical cross-sectional or longitudinal studies preclude an evaluation of nociception-motor interactions and gait compensations that may be attributed to acute pain, as opposed to longer-term factors such as structural changes, chronic pain or learned gait compensations. The acute increase in pain in response to a mechanical stimulus (i.e. weight bearing exercise) presents an opportunity to probe the relationship between gait mechanics and changes in pain in older adults in the absence of changes in disease severity. Prior work quantifying the efficacy of pain pharmacology used a treadmill walking bout to produce an acute pain flare [4, 22]. A similar protocol may be able to discern the contribution to or response of gait mechanics to acute exercise-induced pain flares. Therefore, the aims of this study were to quantify 1 ) the impact of baseline knee joint mechanics and co-activation on changes in OA pain severity in response to a bout of treadmill walking and 2) the biomechanical response to increased pain severity. It was hypothesized that larger knee joint moments and greater muscle co-activation would yield greater pain flares with walking. In addition we hypothesized that there would be increases in perceived pain along with a reduction in the knee flexion angles, peak knee joint moments and an increase in co-activation of muscles crossing the knee joint in response to a bout of treadmill walking.

\section{Methods}

Participants with and without symptomatic knee OA were recruited from surrounding communities via flyer, advertisements and word of mouth. All participants provided written informed consent as approved by the University of Massachusetts-Amherst Internal Review Boards. A power analysis was completed using data from the literature on knee OA gait with pain $[15,23]$ and indicated group sizes of $n=6$ to 12 were needed to detect $10-20 \%$ differences in knee kinematic and kinetics parameters with a power of $\beta=0.8$ and corresponding effect sizes ranging from 0.4-1.5. Inclusion criteria were ages 50-75 years, BMI $<35 \mathrm{~kg} / \mathrm{m}^{2}$, good general health, ability to walk unaided, and no history of cardiovascular or neurological disorders. Participants for the OA group met the American College of Rheumatology clinical classification criteria for $\mathrm{OA}$ in at least 1 knee and reported physician-diagnosed knee OA [24]. Prior to participation in study activities, participants first completed an IRB approved informed consent document and the Physical Activity Readiness Questionnaire for Everyone to assess risk factors for exercise participation. Participants were asked to refrain from taking pain medication for $24 \mathrm{~h}$ prior to their study visit. Knee OA symptom severity and physical function were captured using the Knee Osteoarthritis Outcome Score (KOOS) [25]. Participants then completed a standardized testing protocol that included reporting pain on a verbal numeric rating scale (vNRS), overground gait analysis, self-paced treadmill walk, and a repetition of the pain reporting and overground gait analysis. 


\section{Overground gait analysis}

Participants completed 3 walking trials at preferred pace and then at a faster than preferred pace over a $25 \mathrm{~m}$ walkway while kinematic and kinetic data were collected. For the faster than preferred condition, participants were instructed to walk as if they were "trying to catch a bus". The Point Cluster Technique (PCT) marker set up was used on the OA participants' more affected limb and the right limb for controls [26]. With the PCT marker proto$\mathrm{col}$, clusters of nine and seven reflective markers are distributed on the thigh and shank, respectively. Cluster coordinate systems are determined for the thigh and shank separately by calculating principal axes of the clusters assuming a unit weight for each marker. During a static reference trial, markers placed on bilateral greater trochanter, posterior superior iliac spine, anterior superior iliac spine; medial and lateral femoral epicondyles, tibia plateau, and malleoli; 5th metatarsal head and heel; and the marker clusters establish the tibial, femoral, foot and pelvic anatomic coordinate systems. The relative position and orientation between the marker cluster coordinate systems and the anatomical coordinate systems are calculated in the reference trial. Joint angles are calculated as projected angles and joint moments are calculated via inverse dynamics and reported as external moments, resolved in the distal coordinate system. Due to problems with marker occlusion, gait data could not be used for 1 knee OA and 2 control participants.

\section{Exercise protocol}

Participants completed a 20 min treadmill walk (20MTW) at preferred walking pace. Treadmill speed was started below participants' overground preferred walking speed and then increased or decreased in increments of $0.1 \mathrm{mph}$ until participants indicated that the pace felt normal and could be sustained for $20 \mathrm{~min}$. Perceived pain was evaluated on an 11 point vNRS every two minutes throughout the treadmill walk. The pain ratings in the first and final 2 min of the 20MTW were used to evaluate acute changes in pain in response to exercise.

\section{Directed co-contraction ratio}

Electromyography (EMG, Trigno Delsys, MA, USA) was collected at $2000 \mathrm{~Hz}$ during the second and last minutes of the 20MTW. Electrodes were placed over the rectus femoris, vastus lateralis, vastus medialis, biceps femoris, semitendinosus, medial and lateral gastrocnemii and tibialis anterior according to SENIAM guidelines [27]. Raw EMG data had offset removed, were band-pass filtered $(20-500 \mathrm{~Hz})$, full wave rectified and then filtered with a zero lag, fourth order, $20 \mathrm{~Hz}$ low pass Butterworth filter to create linear envelopes using custom MatLab code. Heelstrike and toe-off were identified using an accelerometer placed on the lower leg. EMG for each muscle was then normalized to the average stance phase activity from 10 strides in the second minute of the 20MTW [28]. Directed co-contraction ratios (DCCR) were calculated to compare relative activation between the knee extensors (rectus femoris and vasti) and knee flexors (hamstrings and gastrocnemii) as well as between lateral (vastus lateralis, biceps femoris, and lateral gastrocnemius) and medial (vastus medialis, semitendinosus, and medial gastrocnemius) knee muscles [29]. The DCCR was calculated at each data point $t$ for each stride $s$ using one of two equations:

For the extensors vs. flexors ratio, if extensor activation was greater than flexor activation:

$$
D C C R_{t, s}=1-\frac{(\text { average of flexor linear envelopes })_{t, s}}{(\text { average of extensor linear envelopes })_{t, s}}
$$

Else

$$
D C C R_{t, s}=\frac{(\text { average of extensor linear envelopes })_{t, s}}{(\text { average of flexor linear envelopes })_{t, s}}-1
$$

The same procedure was followed for the lateral vs. medial ratio with lateral muscles replacing extensors and medial muscles replacing flexors in the above equations. For DCCRs, values closer to 1 or -1 indicate activation that is primarily due to one group in the ratio (for +1 , greater extensor or lateral activation; for -1 , greater flexor or medial activation). Values close to 0 indicate relatively equal activation of both muscle groups in the ratio. DCCRs for the extensor:flexor and lateral:medial comparisons were averaged over terminal swing (last 15\% of swing) and early, mid, and late (thirds of) stance. Due to technical issues during the data collection EMG data for 4 $\mathrm{OA}$ and 3 controls were excluded from the analysis.

\section{Primary outcomes}

The primary outcomes for this study were selected based on proposed pain-induced motor system adaptation strategies [16-18]. These proposed pain adaptations include: an increase in stiffness and decrease in joint movement (knee flexion angles at foot contact, loading response peak and toe-off, and increased co-activation of muscles crossing the knee); a reduction in total load on the painful joint (vertical ground reaction force, knee flexion and internal rotation and total reaction moment); and a redistribution of load across or within the medial and lateral compartments (1st and 2nd peak knee adduction moments, mean knee internal-external rotation angle over stance). The total reaction moment was calculated as the root-mean-square of the three components of the knee joint moments [30]. The total reaction moment is a resultant measure and a surrogate measure of the total load on the medial compartment. Secondary 
outcome measures to gain insight to compensatory strategies in OA and with pain included: ankle and hip flexion angles at heel-strike, ankle and hip range of motion in stance, peak ankle eversion angle, peak hip flexion, extension and 1st peak adduction moment and peak ankle plantar flexion, dorsiflexion and eversion moments.

\section{Statistical analysis}

Preliminary evaluation of the pain changes in response to the 20MTW indicated that not all participants experienced a clinically important difference in pain (i.e., flare). Thus, OA participants were split into pain flare and no flare groups based on changes in vNRS. Participants who reported a change in pain $\geq 1$ point in response to the $20 \mathrm{MTW}$ were assigned to the pain flare group. $\mathrm{A} \geq 1$ point change is considered a minimal clinically important difference on the vNRS for individuals with mild to moderate baseline pain [31, 32]. Un-paired student's t-tests were used to test for differences in participant characteristics and patient reported outcomes between pain flare and no flare OA groups. Two-way ANOVAs $(\alpha=0.05)$ were used to test for significant group, condition (preferred or faster than preferred) and group by condition interaction effects at baseline and for changes in the overground kinematics and kinetics in response to the 20MTW. One-way ANOVAs were used to test for an effect of group for co-activation during the 2nd minute of treadmill walking and the change between the 2nd and 20th minute of treadmill walking. Least significant difference post-hoc analysis was used to quantify pair-wise group differences where main effects were found. Cohen's d effect sizes of the differences were calculated and a medium effect size was considered $d>0.5$ and a large effect $d>0.8$. Of note, an enrollment target of 18 for the OA group was made to power the study to test the hypothesis that the change in pain was significantly different from zero using literature data [4]. However, the pain response of our participants was very different from the prior study and as such, the study is not powered to test for differences in the change in pain with these subgroupings.

\section{Results}

Thirty-six adults (17 healthy older and 19 with mild to moderate symptomatic knee OA) were enrolled in this study. Eight of nineteen OA participants had a significant flare response to the 20MTW. There were no OA group differences in the treadmill speed for the 20MTW ( $p=$ 0.89 ), demographics (age, $p=0.85$ and BMI, $p=0.9$ ) or participant reported OA symptoms (KOOS pain, $p=$ 0.09 and ADL function, $p=0.16$ ) (Table 1).

\section{Baseline}

At baseline, group effects were found for overground walking speed $(p=0.04)$, cadence $(p=0.05)$ heel-strike and toe off knee flexion angles ( $p<0.001$ for both), knee internal-external rotation angle over stance $(p=0.05)$, and heel-strike hip flexion angle $(p=0.001)$ (Table 2 and Additional file 1: Table S1). There were no group by condition interaction effects. Post-hoc comparisons indicated that OA groups walked slower and did not achieve as much knee extension (at heel-strike) or flexion (at toe-off) as healthy controls. In addition, the pain flare group walked with a slower cadence, and a more internally rotated femur relative to the tibia compared to both the no flare and controls.

Knee flexion $(p<0.001)$, 2nd peak knee adduction $(p=0.04)$, knee internal rotation $(p=0.002)$, total knee reaction $(p=0.001)$, hip extension $(\mathrm{p}<0.001)$, 1st peak hip adduction ( $\mathrm{p}=0.001)$, and peak ankle plantar-flexion moments $(p=0.003)$ differed between groups (Tables 3 and 4 and Additional file 2: Table S2). At baseline the no flare

\section{$\Delta$ kinematics with $20 \mathrm{MTW}$}
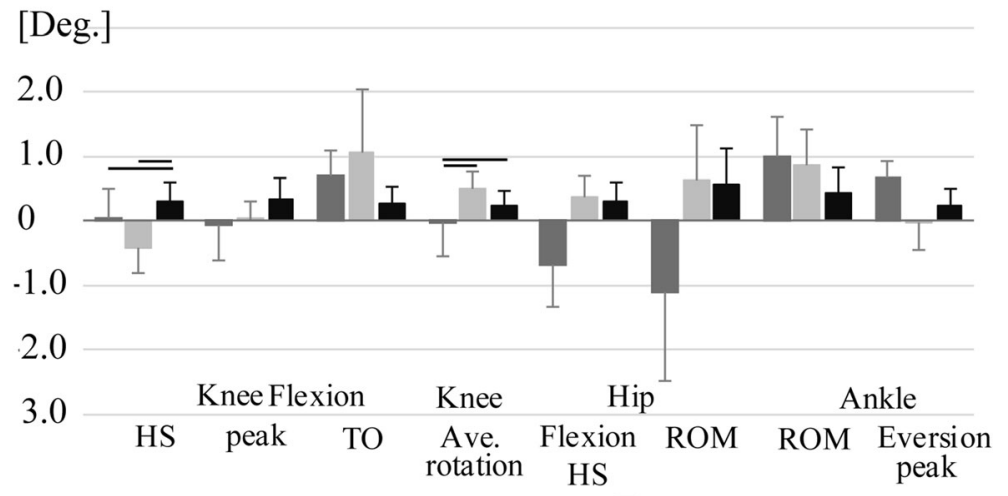

Pain flare No flare Control

Fig. 1 Mean changes $( \pm S E)$ in hip, knee and ankle kinematics with the 20-min treadmill walk (20MTW). Black bars indicate significant post-hoc group differences $a=0.05$ 
Table 1 Group demographics (Mean and SE) including preferred treadmill walking speed and participant reported symptoms at baseline and the change with the 20MTW. vNRS: verbal numerical rating scale pain measure

\begin{tabular}{llllllll}
\hline Normal speed & Age & BMl $\left(\mathrm{kg} / \mathrm{m}^{2}\right)$ & $\begin{array}{l}\text { Treadmill } \\
\text { Speed }(\mathrm{m} / \mathrm{s})\end{array}$ & Baseline vNRS & $\begin{array}{l}\text { Change in } \\
\text { pain (vNRS) }\end{array}$ & KOOS Pain & KOOS ADL \\
\hline Pain Flare $(n=8 ; 3 \mathrm{~F})$ & $62.1(1.9)$ & $25.89(1.47)$ & $0.92(0.13)$ & $2.63(1.00)$ & $1.50(0.27)^{\mathrm{a}}$ & $59.1(7.09)$ & $68.1(8.5)$ \\
No Flare $(n=11 ; 10 \mathrm{~F})$ & $62.6(1.9)$ & $25.69(1.01)$ & $0.90(0.07)$ & $1.36(0.53)$ & $-0.05(0.11)^{\mathrm{a}}$ & $70.7(4.19)$ & $79.3(3.0)$ \\
Controls $(n=17 ; 14 \mathrm{~F})$ & $64.5(1.5)$ & $24.3(0.9)$ & $1.08(0.06)$ & - & - & - & - \\
\hline
\end{tabular}

indicates a significant difference between OA groups

group had smaller knee flexion and total reaction moments compared to both the pain flare and control groups. In comparison to the pain flare group only, the no flare group had smaller peak ankle plantarflexion moments and displayed a medium effect size for a smaller in 2nd peak knee adduction moment. In comparison to the control group only, the no flare group had smaller 2nd peak knee adduction, knee internal rotation moments as well as smaller hip flexion, extension and 1st peak adduction moments. Only the knee internal rotation, hip extension and 1st peak hip adduction moments were smaller for the pain flare as compared to control group. There was a group effect for muscle co-activation (DCCR) in late stance for both muscle group comparisons (Table 5). The flare group displayed greater relative hamstrings (vs. quadriceps) and medial (vs. lateral) activation compared to the controls $(p=0.04, p=0.04)$ and the no flare groups $(p=0.04, p=0.007)$.

\section{Response to 20MTW}

In response to the 20MTW there was a group effect for the change in heel-strike knee flexion angle $(p=0.04)$ and mean knee internal-external rotation angle over stance $(p=0.05)$ (Fig. 1). There was also a condition effect indicating a greater magnitude of change with the 20MTW in the faster than preferred condition for walking speed $(p=0.002)$, cadence $(p=0.02)$, vertical ground reaction force $(p=0.05)$ and hip flexion range of motion $(p=0.002)$. There were no group by condition interaction effects. There was a difference in the change in knee flexion at heel-strike for the pain flare $(p=0.05$, $\mathrm{d}=0.52)$ and no flare $(p=0.03, \mathrm{~d}=0.28)$ as compared to the controls. In addition, the change in average internal tibia rotation with respect to the femur was greater for the pain flare as compared to both no flare and controls $(p=0.02, \mathrm{~d}=0.66 \& p=0.04, \mathrm{~d}=0.41$ respectively). However, the average change in kinematics was less than 1 degree for all outcomes.

In response to the 20MTW there was a main group effect for the change in 2nd peak knee adduction moment $(p=0.05)$, knee internal rotation moment $(p=0.005)$ and ankle eversion moment $(p=0.02)$ and a trend for a group effect for the knee flexion $(p=0.08)$ and 1st peak knee adduction moments $(p=0.1)$. There were no main effects for condition. There was a significant group by condition interaction effect for the hip extension moment. The response to the 20MTW did not differ between the no flare and control group $(p>0.1$ and $\mathrm{d}<0.4$ for all comparison) but did differ between the pain flare and other groups. The pain flare group displayed a larger decrease compared to no flare group for the 1st peak knee adduction moment $(p=0.03, \mathrm{~d}=0.60)$, 2nd peak knee adduction moment $(p=0.02, \mathrm{~d}=0.68)$, knee internal rotation moment $(p=0.002, \mathrm{~d}=0.82)$ and ankle eversion moment ( $p=0.02, \mathrm{~d}=0.59)$ (Fig. 2). The effect size for the difference in changes for pain flare vs no flare was moderate for the knee flexion moment $(p=0.1$; $\mathrm{d}=0.52$ ). In addition, there were greater decreases for the pain flare vs control group for the knee flexion moment $(p=0.03 ; \mathrm{d}=0.66)$, knee internal rotation moment $(p=0.005, \mathrm{~d}=0.82)$ and ankle eversion moment $(p=0.007, \mathrm{~d}=0.73)$. The decreases in the 1 st and $2 \mathrm{nd}$ peak knee adduction moment were on average 3.5 times greater for the pain flare group as compared to the control

Table 2 Baseline knee angles for preferred speed overground walking, Mean (SE)

\begin{tabular}{|c|c|c|c|c|c|c|}
\hline & $\begin{array}{l}\text { Overground speed }{ }^{a} \\
(\mathrm{~m} / \mathrm{s})\end{array}$ & $\begin{array}{l}\text { Cadence }^{a} \\
\text { (step/min) }\end{array}$ & $\begin{array}{l}\text { Heel-strike Flexion }{ }^{\text {a }} \\
\text { (deg) }\end{array}$ & $\begin{array}{l}\text { Peak Flexion } \\
\text { (deg) }\end{array}$ & $\begin{array}{l}\text { Toe-off Flexion }{ }^{a} \\
\text { (deg) }\end{array}$ & $\begin{array}{l}\text { Average In/ext. rotation } \\
\text { (deg) }\end{array}$ \\
\hline Pain Flare $(n=8)$ & $1.35(0.10)$ & $58.28(1.51)$ & $9.71(2.76)$ & $20.49(2.44)$ & $58.25(3.50)$ & $3.07(1.20)$ \\
\hline No Flare $(n=11)$ & $1.35(0.06)$ & $59.41(0.90)$ & $6.70(1.31)$ & $16.06(1.68)$ & $62.33(1.97)$ & $-1.47(1.63)$ \\
\hline Controls $(n=15)$ & $1.48(0.03)$ & $60.43(0.97)$ & $1.66(1.14)$ & $18.64(1.15)$ & $67.36(0.91)$ & $-0.04(1.25)$ \\
\hline \multicolumn{7}{|l|}{$p$-value /effect size } \\
\hline PF vs C & $p=0.04 d=0.83$ & $p=0.02 d=0.78$ & $p<0.001 \mathrm{~d}=1.48$ & & $p<0.001 \mathrm{~d}=1.51$ & $p=0.05 d=0.70$ \\
\hline NF vs C & $p=0.09 d=0.74$ & & $p=0.002 \mathrm{~d}=1.00$ & & $p=0.002 d=0.98$ & \\
\hline PF vs NF & & $p=0.05 d=0.60$ & & & & $p=0.02 \mathrm{~d}=0.95$ \\
\hline
\end{tabular}

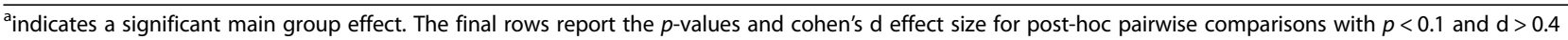


Table 3 Baseline external knee moments (\%BW x Ht) for preferred speed overground walking, Mean (SE)

\begin{tabular}{|c|c|c|c|c|c|c|}
\hline & $\begin{array}{l}\text { 1st Peak vertical } \\
\text { GRF BW }\end{array}$ & Flexion $^{a}$ & 1st Adduction & 2nd Adduction ${ }^{a}$ & Internal rotation ${ }^{a}$ & Total reaction ${ }^{a}$ \\
\hline Pain Flare $(n=7)$ & $1.16(0.03)$ & $3.65(0.58)$ & $-2.94(0.34)$ & $-1.95(0.32)$ & $-0.76(0.13)$ & $4.85(0.46)$ \\
\hline No Flare $(n=11)$ & $1.14(0.04)$ & $2.30(0.39)$ & $-2.73(0.29)$ & $-1.44(0.24)$ & $-0.73(0.09)$ & $3.73(0.34)$ \\
\hline Controls $(n=15)$ & $1.22(0.02)$ & $4.10(0.31)$ & $-3.33(0.33)$ & $-2.04(0.31)$ & $-1.03(0.09)$ & $5.38(0.37)$ \\
\hline \multicolumn{7}{|l|}{$p$-value/effect size } \\
\hline PF vs $C$ & & & & & $p=0.03 d=0.66$ & \\
\hline NF vs C & & $p<0.001, d=1.24$ & & $p=0.01, d=0.67$ & $p=0.001 \mathrm{~d}=1.00$ & $p<0.001, d=1.12$ \\
\hline PF vs NF & & $p=0.02 \mathrm{~d}=0.84$ & & $p=0.1 d=0.7$ & & $p=0.05 d=0.84$ \\
\hline
\end{tabular}

group however the large variance between participants in the change led to moderate effect sizes $(p=0.1, \mathrm{~d}=$ $0.49 ; p=0.9 ; \mathrm{d}=0.46)$. There were no differences in the magnitude of change in DCCR for either muscle grouping (Table 5).

\section{Discussion}

The aim of this study was to quantify the impact of walking mechanics and muscle co-activation on changes in OA pain with a bout of exercise and then to examine the biomechanical response to increased pain. In response to the 20MTW almost half of the OA participants reported clinically relevant increases in pain. Those OA participants who experienced a pain flare had greater joint moments at baseline as compared to those who did not experience a pain flare and greater muscle co-activation as compared to both no flare and control groups. This suggests that individuals with OA prone to exercise-induced pain flares have greater joint loads and are more similar to healthy controls as compared to individuals with OA who do not experience pain flares. In response to the 20MTW, there were significantly greater changes in gait mechanics for the pain flare group as compared to both control and no flare OA groups. Thus despite baseline gait that may increase risk for pain, individuals who experienced a pain flare are able to rapidly adapt their mechanics to small variations in joint pain. These results also suggest that individuals who are more susceptible to these pain flares may experience the greatest benefits of biomechanical intervention such as variable stiffness shoes [33] or gait retraining [34, 35].

Beyond the observations of increased pain with activity as well as greater pain with faster as compared to slower walking $[4,22]$, there is limited literature on the role of in-vivo gait mechanics on pain in OA. Supporting a key role of gait mechanics in the OA pain experience, the individuals who did not experience a pain flare tended to have smaller magnitude joint moments at the knee as compared to the controls and OA participants who experienced pain flares. In addition, for those individuals who experienced a flare, the knee flexion and adduction moments, surrogate markers for the loading at the knee joint, were not different compared to controls and there

Table 4 Baseline values for the hip and ankle outcome measures at the preferred walking pace. The last row reports the $p$-values and cohen's $d$ effect size for post-hoc pairwise comparisons with $p<0.1$ and $d>0.4$

\begin{tabular}{|c|c|c|c|c|c|c|c|c|c|c|}
\hline & \multicolumn{2}{|l|}{$\begin{array}{l}\text { Hip Angle } \\
\text { (deg) }\end{array}$} & \multicolumn{2}{|c|}{$\begin{array}{l}\text { Ankle Angles } \\
\text { (deg) }\end{array}$} & \multicolumn{3}{|l|}{$\begin{array}{l}\text { Hip Moments } \\
\left(\% \mathrm{BW}^{*} \mathrm{Ht}\right)\end{array}$} & \multicolumn{3}{|l|}{$\begin{array}{l}\text { Ankle Moments } \\
\left(\% \mathrm{BW} *^{*} \mathrm{Ht}\right)\end{array}$} \\
\hline & $\begin{array}{l}\text { Heel-strike } \\
\text { flexion }\end{array}$ & $\begin{array}{l}\text { Flexion } \\
\text { ROM }\end{array}$ & $\begin{array}{l}\text { Flexion } \\
\text { ROM }\end{array}$ & $\begin{array}{l}\text { Peak } \\
\text { Eversion }\end{array}$ & Flexion & Extension & 1st Adduction & PlantarFlexion & $\begin{array}{l}\text { Dorsi- } \\
\text { Flexion }\end{array}$ & Eversion \\
\hline Pain Flare & 43.70 & 42.06 & 26.19 & 2.56 & -4.40 & 3.84 & -5.52 & 1.75 & -7.99 & 0.84 \\
\hline SE & 1.77 & 3.10 & 2.29 & 1.33 & 0.70 & 0.37 & 0.53 & 0.13 & 0.61 & 0.29 \\
\hline No Flare & 38.28 & 45.78 & 28.78 & 6.05 & -3.20 & 4.10 & -5.73 & 1.22 & -8.58 & 0.58 \\
\hline SE & 2.00 & 2.16 & 2.24 & 1.09 & 0.37 & 0.22 & 0.31 & 0.11 & 0.26 & 0.10 \\
\hline Control & 34.51 & 47.37 & 29.91 & 4.03 & -4.53 & 5.41 & -6.74 & 1.91 & -8.90 & 0.55 \\
\hline SE & 2.01 & 1.11 & 1.16 & 0.76 & 0.27 & 0.31 & 0.21 & 0.18 & 0.26 & 0.08 \\
\hline \multicolumn{11}{|c|}{$p$-value/effect size } \\
\hline \multicolumn{3}{|l|}{ PF Vs C } & & & & $p<0.001, d=1.10$ & $p<0.001, d=1.08$ & & & \\
\hline \multicolumn{3}{|l|}{ NP vs C } & & & $p<0.001 \mathrm{~d}=0.77$ & $p<0.001, d=0.90$ & $p<0.001, d=1.02$ & & & \\
\hline \multicolumn{3}{|l|}{ PF vs NF } & & & & & & \multicolumn{3}{|l|}{$p=0.04 \mathrm{~d}=1.40$} \\
\hline
\end{tabular}


Table 5 Baseline and change in DCCR for the quadriceps: hamstrings and medial: lateral muscle grouping

\begin{tabular}{|c|c|c|c|c|c|c|c|c|c|}
\hline & & \multicolumn{4}{|c|}{ Quadriceps: Hamstrings } & \multicolumn{4}{|l|}{ Medial: Lateral } \\
\hline & & Terminal Swing & Early Stance & Mid stance & $\overline{\text { Late Stance }}$ & Terminal Swing & Early Stance & Mid stance & $\overline{\text { Late Stance }}$ \\
\hline \multirow[t]{6}{*}{ Baseline } & Pain flare & -0.37 & 0.12 & -0.04 & $-0.26^{*}$ & -0.02 & 0.01 & 0.06 & $-0.15^{*}$ \\
\hline & SE & 0.1 & 0.04 & 0.09 & 0.08 & 0.11 & 0.03 & 0.06 & 0.07 \\
\hline & No flare & -0.29 & 0.09 & 0.00 & $0.04^{*}$ & -0.04 & -0.03 & -0.02 & $0.1^{*}$ \\
\hline & SE & 0.09 & 0.03 & 0.04 & 0.09 & 0.07 & 0.03 & 0.05 & 0.04 \\
\hline & Controls & -0.42 & 0.04 & 0.04 & $0.02^{*}$ & 0.01 & 0.01 & -0.04 & $0.03^{*}$ \\
\hline & SE & 0.04 & 0.03 & 0.05 & 0.06 & 0.07 & 0.02 & 0.04 & 0.04 \\
\hline \multirow[t]{6}{*}{ Change with 20MTW } & Pain flare & 0.00 & -0.12 & 0.19 & 0.11 & -0.08 & -0.08 & 0.09 & 0.13 \\
\hline & SE & 0.07 & 0.09 & 0.08 & 0.09 & 0.06 & 0.06 & 0.06 & 0.06 \\
\hline & No flare & 0.07 & -0.06 & -0.07 & -0.03 & -0.01 & -0.01 & 0.13 & 0.07 \\
\hline & SE & 0.11 & 0.08 & 0.05 & 0.07 & 0.07 & 0.07 & 0.09 & 0.06 \\
\hline & Controls & 0.13 & 0.06 & 0.12 & 0.13 & -0.04 & -0.04 & -0.04 & -0.01 \\
\hline & SE & 0.08 & 0.06 & 0.09 & 0.11 & 0.05 & 0.05 & 0.04 & 0.06 \\
\hline
\end{tabular}

* indicates $p<0.05$ for the pain compared to other groups

was greater co-activation of the medial compared to lateral musculature despite the pain flare group walking at a slower speed than the controls. Together this suggests greater medial joint loading before the onset of pain in this flare group. The lack of a difference between OA pain flare and control groups is somewhat surprising as we might expect those with more severe symptoms in the flare group to have adapted their gait to a greater degree. However, the greater change in pain with a 20MTW for individuals with greater joint moments fits with initial evidence from studies examining load modifying shoe interventions that indicates a reduction in the external knee adduction moment can lead to moderate pain relief $[15,36]$. Together these findings suggest that "poor" mechanics resulting in greater joint loading may contribute to development of pain during weight bearing activity. Those individuals with OA who have not adapted their gait to reduce loading as compared to healthy older adults may be at greater risk for pain flares but may also be at greater risk for disease progression [37].

Individuals with knee pain are hypothesized to adopt neuromuscular strategies to increase knee joint stiffness, decrease joint movement and alter movement patterns to shift or reduce tissue loads and thus limit pain [18]. The dominant response to increased pain in the OA flare group was a reduction in the magnitude of loading via a reduction and redistribution of loads through decreases in knee joint moments. For the pain flare group

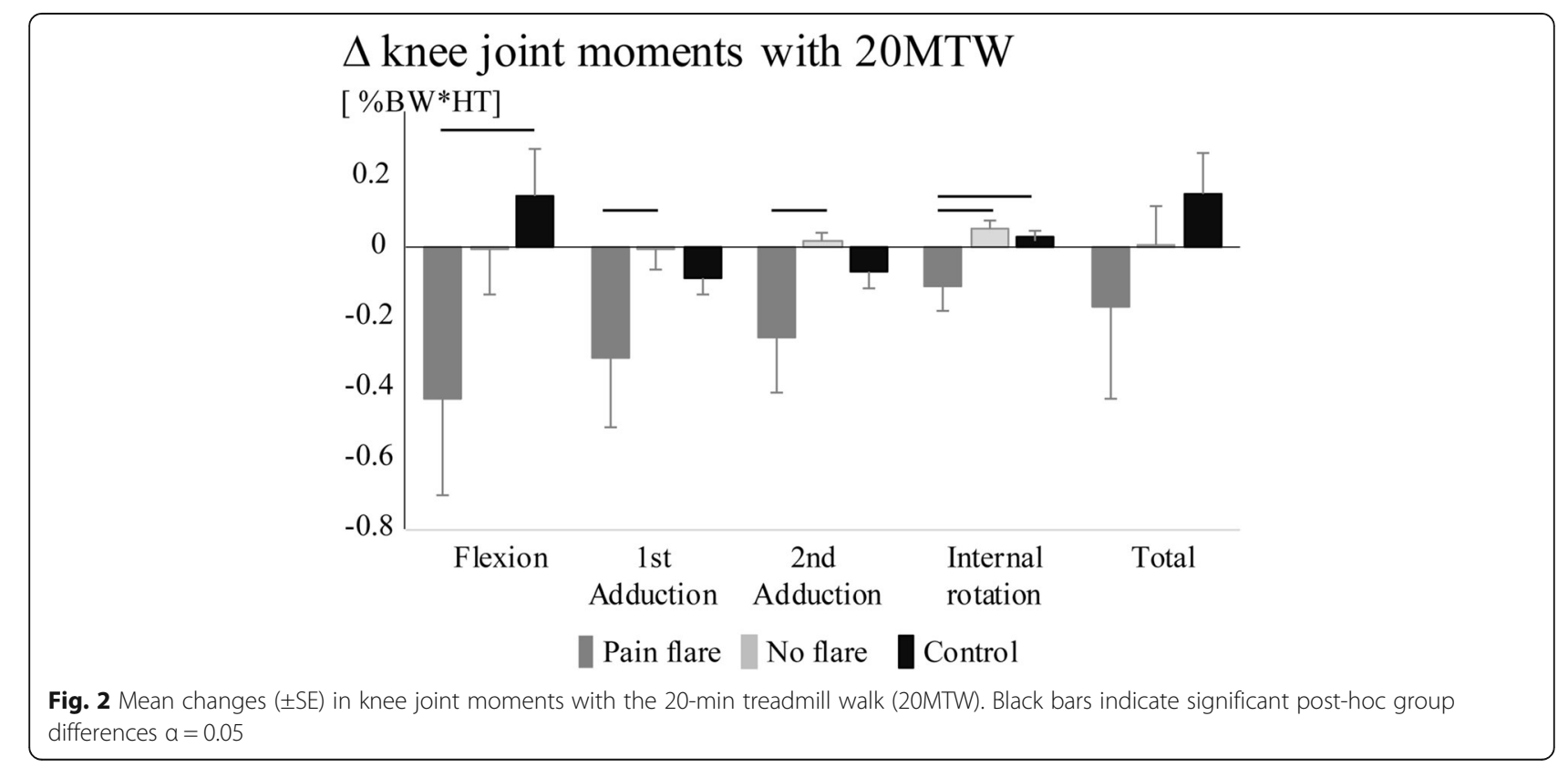


there were significantly greater decreases in the peak knee flexion, 1st and 2nd peak knee adduction and peak internal rotation moments as compared to the no flare or control groups with the 20MTW. The changes in peak knee flexion and first peak knee adduction moments were on the order of $12-15 \%$ for the flare group and $0-4 \%$ for the other groups. This response in the pain group is consistent with the response to experimental pain models that produce acute pain in a healthy joint using an intra-articular injection of hypertonic saline [19]. These results suggest that, even in the presence of baseline pain, the motor system in OA remains highly sensitive and able to adapt on a short time scale to small magnitude variations in OA-related joint pain that may occur over a day or a week's time $[3,38]$.

For the pain flare group the magnitude of the pain change (vNRS $\sim 1.5$ points) was smaller than in a prior study using a similar walking protocol in patients with moderate to severe OA [4]. This may be expected as the current participants, in contrast to the previous, had less severe pain at baseline (vNRS $\sim 1.89$ points), reported a lesser impact of OA pain and symptoms in daily life and were not required to report an exacerbation of pain prior to enrollment. Additional support for this impact of baseline symptoms on the evoked pain flare magnitude is provided by the tendency for the participants in the OA pain flare group to have greater symptom severity as compared to the no flare group. Greater baseline pain may be indicative of greater inflammation or bony pathology and thus a greater mechano-sensitivity of the tissue [39] during walking. Participants in this study were recruited to have mild to moderate but not severe $\mathrm{OA}$, as the potential to intervene and improve OA-related quality of life for a significant number of years using exercise or biomechanical interventions is the greatest in this population.

Why some OA participants may have adapted their gait to off-load the diseased joint while others have not is not clear. Understanding the factors that contribute to a beneficial gait adaptation at baseline may facilitate efforts to improve exercise adherence for symptomatic OA patients. The off-loading response to the pain increase with the treadmill walk suggests the motor system still responds in the expected way to a pain stimulus, despite the lack of adaptation at baseline in these individuals. However, a greater variance in biomechanical response in the flare group may indicate that there are some subject-specific adaptation strategies to increased OA-related pain. Of note, there were not differences in the DCCR following the 20MTW, a surprising finding given that prior experimental pain work shows a significant inhibition of the knee extensors with pain [40]. This may be due to limitations in the DCCR metric but may also be true differences in the motor system response to fluctuations in chronic pain versus the onset of acute experimental pain. As with most co-contraction metrics, the DCCR only quantifies the relative activation of the selected muscles. However there was not a change in the net activations (i.e. value of the numerator or denominator) following the 20MTW for any group.

While patient reported symptomatic severity of knee OA was captured, a key limitation of this study is a lack of documentation of the OA structural severity in the participants. Magnetic resonance imaging to document the presence and severity of cartilage thinning and pain-producing pathology such as synovitis, bone marrow lesions or meniscal damage [41, 42] may provide insight to why some individuals experience greater pain with exercise. Specifically, differences in the type or location of pain-producing pathologies such as bone marrow lesions [43] or more lateral vs medial compartment disease may impact both the magnitude of an exercise induced pain flare and the biomechanical response to increased pain. Additional studies to investigate the potential for variations in knee extensor muscle function, structural severity, bone marrow lesions or synovitis to impact the gait response are warranted. In this study, we only examined a single exercise stimulus, moderate walking. To translate these finding to the general public, further study is needed to determine the mechanical loading characteristics that have the greatest impact on exercise-induced pain and to quantify the biomechanical response for a broader range of activities of daily living such as balance, stair ascent and descent and rising from a chair.

\section{Conclusions}

Exercise induced pain flares represent a significant barrier for individuals with $\mathrm{OA}$ to meet recommendations for physical activity and adhere to exercise interventions. The results of this study suggest that individuals who have adapted their gait to reduce knee joint loads may be less susceptible to exercise induced pain flares. This highlights a potential role of gait biomechanics in short term OA pain fluctuations. The study findings also suggest that despite the chronic nature of OA pain, OA gait patterns are not fixed and the motor system's ability to respond to nociceptive stimuli in $\mathrm{OA}$ remains intact. The resulting changes in joint loading due to periodic fluctuations in pain level may have both beneficial or detrimental cartilage health and long-term OA outcomes and thus should not be ignored.

\section{Additional files}

Additional file 1: Table S1. Baseline knee angles for Faster than preferred speed overground walking. ${ }^{*}$ indicates a significant main group effect. (DOCX 68 kb) 
Additional file 2: Table S2. Baseline external knee moments (\%BW $x$ $\mathrm{Ht}$ ) for Faster than preferred speed overground walking. * indicates a significant group effect. (DOCX $63 \mathrm{~kb})$

\section{Abbreviations}

20MTW: 20 min treadmill walk; DCCR: Directed co-contraction ratio;

EMG: Electromyography; OA: Osteoarthritis; vNRS: Verbal numeric rating scale

\section{Acknowledgements}

The authors thank Carl Jewell for assistance in data collection.

\section{Funding}

This study was funded by a University of Massachusetts -Amherst Faculty Research Grant. The funding body did not play a role in the design of the study, collection/analysis/interpretation of data, or in writing the manuscript.

\section{Availability of data and materials}

The datasets generated during the current study will be made available from the corresponding author on reasonable request.

\section{Authors' contributions}

Substantial contributions were made to the study conception and design by $K A B$, acquisition of data by JFH, KAB and analysis and interpretation of data by JFH, KAB. Both authors (KAB, JFH) were involved in drafting the article or revising it critically for important intellectual content, and approved the final version to be submitted for publication. KAB had full access to all of the data in the study and takes responsibility for the integrity of the data and the accuracy of the data analysis.

\section{Ethics approval and consent to participate}

Approval from the University of Massachusetts-Amherst Institutional Review Board, Federal Wide Assurance \# 00003909, was obtained for the protocol as reported in this manuscript.

\section{Consent for publication}

Not applicable.

\section{Competing interests}

The authors declare that they have no competing interests.

\section{Publisher's Note}

Springer Nature remains neutral with regard to jurisdictional claims in published maps and institutional affiliations.

\section{Author details}

${ }^{1}$ Department of Kinesiology, University of Massachusetts-Amherst, 110 Totman Building 30 Eastman Lane, Amherst, MA 01003, USA. ${ }^{2}$ University of Massachusetts-Amherst, Mechanical and Industrial Engineering, Amherst, USA. ${ }^{3}$ Department of Orthopedics and Physical Rehabilitation, University of Massachusetts Medical School, Worcester, USA. ${ }^{4}$ School of Kinesiology, University of Michigan, Central Campus Recreation Building, 401 Washtenaw Avenue, Ann Arbor, Ml 48109, USA.

\section{Received: 5 October 2018 Accepted: 5 March 2019}

\section{Published online: 14 March 2019}

\section{References}

1. Ma VY, Chan L, Carruthers KJ. Incidence, prevalence, costs, and impact on disability of common conditions requiring rehabilitation in the United States: stroke, spinal cord injury, traumatic brain injury, multiple sclerosis, osteoarthritis, rheumatoid arthritis, limb loss, and back pain. Arch Phys Med Rehabil. 2014;95(5):986-995.e1.

2. McAlindon TE, Bannuru RR, Sullivan MC, Arden NK, Berenbaum F, BiermaZeinstra SM, et al. OARSI guidelines for the non-surgical management of knee osteoarthritis. Osteoarthr Cartil. 2014;22(3):363-88.

3. Focht $B C$, Ewing $V$, Gauvin $L$, Rejeski WJ. The unique and transient impact of acute exercise on pain perception in older, overweight, or obese adults with knee osteoarthritis. Ann Behav Med. 2002;24(3):201-10.
4. Peeva E, Beals CR, Bolognese JA, Kivitz AJ, Taber L, Harman A, et al. A walking model to assess the onset of analgesia in osteoarthritis knee pain. Osteoarthr Cartil. 2010;18(5):646-53.

5. Sandal LF, Roos EM, Bogesvang SJ, Thorlund JB. Pain trajectory and exerciseinduced pain flares during 8 weeks of neuromuscular exercise in individuals with knee and hip pain. Osteoarthr Cartil 2016 04;24(4):589-592.

6. Cruz-Almeida Y, Cardoso J, Riley JL 3rd, Goodin B, King CD, Petrov M, et al. Physical performance and movement-evoked pain profiles in communitydwelling individuals at risk for knee osteoarthritis. Exp Gerontol. 2017;98:186-91.

7. Mundermann A, Dyrby CO, Hurwitz DE, Sharma L, Andriacchi TP. Potential strategies to reduce medial compartment loading in patients with knee osteoarthritis of varying severity: reduced walking speed. Arthritis Rheum 2004 04:50(4):1172-1178

8. Astephen JL, Deluzio KJ, Caldwell GE, Dunbar MJ. Biomechanical changes at the hip, knee, and ankle joints during gait are associated with knee osteoarthritis severity. J Orthop Res. 2008;26(3):332-41.

9. Kaufman KR, Hughes C, Morrey BF, Morrey M, An KN. Gait characteristics of patients with knee osteoarthritis. J Biomech. 2001;34(7):907-15.

10. Boyer KA. Biomechanical response to osteoarthritis pain treatment may impair long-term efficacy. Exerc Sport Sci Rev. 2018;46(2):121-8.

11. Maly MR, Costigan PA, Olney SJ. Mechanical factors relate to pain in knee osteoarthritis. Clin Biomech (Bristol, Avon). 2008;23(6):796-805.

12. Hall M, Bennell KL, Wrigley TV, Metcalf BR, Campbell PK, Kasza J, et al. The knee adduction moment and knee osteoarthritis symptoms: relationships according to radiographic disease severity. Osteoarthr Cartil. 2017;25(1):34-41

13. Astephen JL, Deluzio KJ, Caldwell GE, Dunbar MJ, Hubley-Kozey CL. Gait and neuromuscular pattern changes are associated with differences in knee osteoarthritis severity levels. J Biomech. 2008;41(4):868-76.

14. Birmingham TB, Marriott KA, Leitch KM, Moyer RF, Lorbergs AL, Walton DM, et al. Association between knee load and pain: within-patient, betweenknees, case-control study in patients with knee osteoarthritis. Arthritis Care Res (Hoboken). 2018.

15. Variable-stiffness walking shoe lowers knee adduction moment, reduces pain, and improves function in patients with osteoarthritis after one year. ; 2008/03; ; 2008.

16. Lund JP, Donga R, Widmer CG, Stohler CS. The pain-adaptation model: a discussion of the relationship between chronic musculoskeletal pain and motor activity. Can J Physiol Pharmacol. 1991;69(5):683-94.

17. Roland MO. A critical review of the evidence for a pain-spasm-pain cycle in spinal disorders. Clin Biomech(Bristol, Avon) 1986 05;1(2):102-109.

18. Hodges PW, Tucker K. Moving differently in pain: a new theory to explain the adaptation to pain. Pain. 2011;152(3 Suppl):S90-8.

19. Henriksen M, Graven-Nielsen T, Aaboe J, Andriacchi TP, Bliddal H. Gait changes in patients with knee osteoarthritis are replicated by experimental knee pain. Arthritis Care Res (Hoboken). 2010;62(4):501-9.

20. Hug F, Hodges PW, Tucker K. Task dependency of motor adaptations to an acute noxious stimulation. J Neurophysiol. 2014;111(11):2298-306.

21. Hodges PW, Mellor R, Crossley K, Bennell K. Pain induced by injection of hypertonic saline into the infrapatellar fat pad and effect on coordination of the quadriceps muscles. Arthritis Rheum. 2009;61(1):70-7.

22. Farrokhi S, Jayabalan P, Gustafson JA, Klatt BA, Sowa GA, Piva SR. The influence of continuous versus interval walking exercise on knee joint loading and pain in patients with knee osteoarthritis. Gait Posture. 2017:56:129-33.

23. Asay JL, Boyer KA, Andriacchi TP. Repeatability of gait analysis for measuring knee osteoarthritis pain in patients with severe chronic pain. J Orthop Res. 2013;31(7):1007-12.

24. Altman R, Asch $\mathrm{E}$, Bloch D, Bole G, Borenstein D, Brandt $K$, et al. Development of criteria for the classification and reporting of osteoarthritis. Classification of osteoarthritis of the knee. Diagnostic and therapeutic criteria Committee of the American Rheumatism Association. Arthritis Rheum. 1986;29(8):1039-49.

25. Roos EM, Lohmander LS. The knee injury and osteoarthritis outcome score (KOOS): from joint injury to osteoarthritis. Health Qual. Life Outcomes. 2003:1:64

26. Andriacchi TP, Alexander EJ, Toney MK, Dyrby C, Sum J. A point cluster method for in vivo motion analysis: applied to a study of knee kinematics. Biomech Eng. 1998;120(6):743-9.

27. Stegeman DF, Hermens HJ. Standards for surface electromyography: the European project (SENIAM). In: Hermens HJ, Rau G, Disselhorst-Klug C, 
Freriks B, editors. Surface electromyography application areas and parameters. Germany: Workshop on surface electromyography Aachen; 1998. p. 108-12.

28. Burden A, Bartlett R. Normalisation of EMG amplitude: an evaluation and comparison of old and new methods. Med Eng Phys. 1999;21(4):247-57.

29. Heiden TL, Lloyd DG, Ackland TR. Knee joint kinematics, kinetics and muscle co-contraction in knee osteoarthritis patient gait. Clin Biomech (Bristol , Avon ). 2009:09/16.

30. Boyer KA, Angst MS, Asay J, Giori NJ, Andriacchi TP. Sensitivity of gait parameters to the effects of anti-inflammatory and opioid treatments in knee osteoarthritis patients. J Orthop Res. 2012;30(7):1118-24.

31. Salaffi F, Stancati A, Silvestri CA, Ciapetti A, Grassi W. Minimal clinically important changes in chronic musculoskeletal pain intensity measured on a numerical rating scale. Eur J Pain. 2004 Aug;8(4):283-91.

32. Tubach F, Ravaud P, Baron G, Falissard B, Logeart I, Bellamy N, et al. Evaluation of clinically relevant states in patient reported outcomes in knee and hip osteoarthritis: the patient acceptable symptom state. Ann Rheum Dis. 2005;64(1):34-7.

33. Erhart JC, Mundermann A, Elspas B, Giori NJ, Andriacchi TP. A variablestiffness shoe lowers the knee adduction moment in subjects with symptoms of medial compartment knee osteoarthritis. J Biomech 2008 08/ 28:41(12):2720-2725

34. Hunt MA, Schache AG, Hinman RS, Crossley KM. Varus thrust in medial knee osteoarthritis: quantification and effects of different gait-related interventions using a single case study. Arthritis Care Res (Hoboken). 2011; 63(2):293-7.

35. Cheung RTH, Ho KKW, Au IPH, An WW, Zhang JHW, Chan ZYS, et al. Immediate and short-term effects of gait retraining on the knee joint moments and symptoms in patients with early tibiofemoral joint osteoarthritis: a randomized controlled trial. Osteoarthr Cartil. 2018;26(11): 1479-86.

36. Parkes MJ, Maricar N, Lunt M, LaValley MP, Jones RK, Segal NA, et al. Lateral wedge insoles as a conservative treatment for pain in patients with medial knee osteoarthritis: a meta-analysis. JAMA. 2013;310(7):722-30.

37. Chehab EF, Favre J, Erhart-Hledik JC, Andriacchi TP. Baseline knee adduction and flexion moments during walking are both associated with 5 year cartilage changes in patients with medial knee osteoarthritis. Osteoarthr Cartil. 2014 Nov;22(11):1833-9.

38. Allen KD, Coffman CJ, Golightly YM, Stechuchak KM, Keefe FJ. Daily pain variations among patients with hand, hip, and knee osteoarthritis. Osteoarthr Cartil 2009 10;17(10):1275-1282.

39. McDougall JJ. Pain and OA. J MusculoskeletNeuronallnteract 2006 10;6(4): 385-386.

40. Tucker KJ, Hodges PW. Motoneurone recruitment is altered with pain induced in non-muscular tissue. Pain 2009 01;141(1-2):151-155.

41. Hunter DJ, McDougall JJ, Keefe FJ. The symptoms of osteoarthritis and the genesis of pain. Rheum Dis Clin N Am. 2008;34(3):623-43.

42. Baker K, Grainger A, Niu J, Clancy M, Guermazi A, Crema M, et al. Relation of synovitis to knee pain using contrast-enhanced MRIs. Ann Rheum Dis. 2010:05/14.

43. Felson DT, Chaisson CE, Hill CL, Totterman SM, Gale ME, Skinner KM, et al. The association of bone marrow lesions with pain in knee osteoarthritis. Ann Intern Med. 2001;134(7):541-9.

\section{Ready to submit your research? Choose BMC and benefit from:}

- fast, convenient online submission

- thorough peer review by experienced researchers in your field

- rapid publication on acceptance

- support for research data, including large and complex data types

- gold Open Access which fosters wider collaboration and increased citations

- maximum visibility for your research: over $100 \mathrm{M}$ website views per year

At $\mathrm{BMC}$, research is always in progress.

Learn more biomedcentral.com/submissions 